\title{
Tendencias en el consumo de faunas en el piedemonte oriental de la sierra de Velasco (departamento de Castro Barros, La Rioja) - siglos III al X d.C.
}

\author{
Enrique Garate \\ Recibido 18 de mayo 2021. Aceptado 10 de octubre 2021
}

\begin{abstract}
RESUMEN
En este trabajo se presentan los resultados del análisis arqueofaunístico de diferentes sitios de la vertiente oriental de la sierra de Velasco, con el fin de determinar los patrones de consumo en sitios residenciales y productivos a lo largo del primer milenio d.C. Especial énfasis se puso en la explotación de camélidos y las características de su procesamiento por parte de los antiguos habitantes locales, dada la relevancia que estos taxones tuvieron en la región. Asimismo, estos estudios permitieron contrastar hipótesis previas respecto de las actividades desarrolladas en cada sitio. De esta manera, se observó una continuidad en los patrones de consumo a lo largo del período analizado, a la vez que se identificaron diferencias vinculadas a las funcionalidades de cada sitio.
\end{abstract}

Palabras clave: Zooarqueología; La Rioja; Consumo; Camélidos; Unidades domésticas.

\section{Trends in the consumption of fauna in the eastern foothills of the Sierra de Velasco, Department of Castro Barros, La Rioja, third to tenth century AD.}

\begin{abstract}
This paper presents the results of the archaeofaunistic analysis of sites on the eastern slope of the Sierra de Velasco to determine consumption patterns in residential and productive sites throughout the first millennium AD. Special emphasis was placed on the exploitation and processing characteristics of camelids by the former inhabitants, given the importance of these taxa in the region. Likewise, a contrast was made with previous hypotheses regarding activities on each site. A continuity in consumption patterns was observed throughout the analyzed period, while differences linked to the functions of each site were also identified.
\end{abstract}

Keywords: Zooarchaeology; La Rioja; Consumption; Camelids; Domestic units.

\section{INTRODUCCIÓN}

Las primeras ocupaciones humanas en el sector oriental de la sierra de Velasco (La Rioja) (Figura 1) fueron identificadas entre los años 300 y 600 d.C., cuando se produjo la instalación de grupos sedentarios con una estructura de base familiar, descentralizada y con escasa desigualdad en la zona del piedemonte. Un segundo momento en la secuencia ocupacional del área fue determinado entre el 600 y el 900 d.C., en el que se observa un crecimiento de las dimensiones y complejidad arquitectónica. Se ha propuesto que por entonces se configuraron estructuras aldeanas comunitarias, con procesos de integración residencial e interacción regional.

Enrique Garate. Instituto de Ciencias Humanas, Sociales y Ambientales, Consejo Nacional de Investigaciones Científicas y Tecnológicas (INCIHUSA, CONICET). Av. Ruiz Leal s/n Parque General San Martín, Mendoza. Argentina. Email: egarate@mendoza-conicet.gob.ar

Intersecciones en Antropología 22(2), julio-diciembre: 237-248. 2021. ISSN-e 1850-373X

https://doi.org/10.37176/iea.22.2.2021.635

Facultad de Ciencias Sociales - UNICEN - Argentina 
Entre el 900 d.C. y el 1400 d.C., aproximadamente, se produciría una reconfiguración del espacio, que habría dado lugar a nuevos sitios residenciales emplazados en sectores más elevados, en torno al inicio de las quebradas (Cahiza, 2015; Sabatini, 2019; Cahiza et al., 2021).

Hasta el momento, las tareas arqueológicas realizadas en el área integraron estudios arqueofaunísticos que enfatizaron en la identificación de especies, con el fin de caracterizar los patrones de subsistencia en determinados sitios y buscando definir las prácticas cotidianas desarrolladas en ellos. Las primeras investigaciones locales fueron Ilevadas a cabo por Raviña y Callegari (1988, 1992) en la localidad de Anillaco; por Mercado (1993) en Anjullón; y más adelante, por Ortiz Malmierca (2001) y Dlugosz et al. (2009) en la localidad de Chuquis. Sus principales objetivos fueron la determinación cronoestratigráfica, los patrones de asentamiento, la realización de estudios paleobotánicos y la definición de estilos cerámicos.

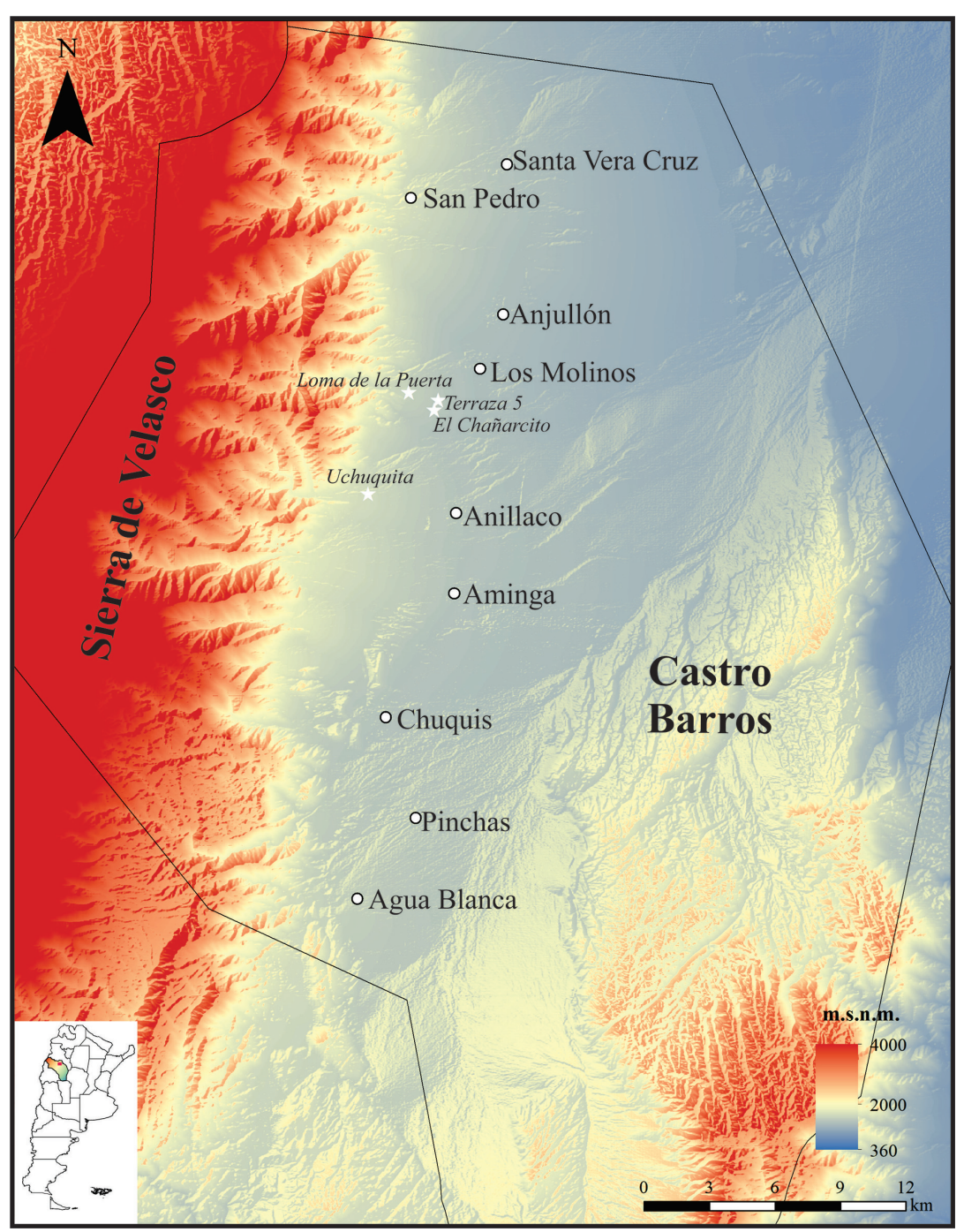

Figura 1- Localización del área de estudio y los sitios analizados.
Los análisis zooarqueológicos realizados por esos autores destacaron el consumo de camélidos (posiblemente domesticados) y de algunos taxones menores como Microcavia, chinchillas, vizcachas y huevos de suri. Respecto de los camélidos, Raviña y Callegari (1992) han señalado que las pasturas disponibles en los sectores superiores de la sierra habrían hecho posible la realización de prácticas de pastoreo.

En este trabajo se pretende integrar los estudios del registro óseo de sitios con diversa caracterización temporal, arquitectónica y funcional, con el objetivo de determinar patrones de consumo y procesamiento animal, contrastando estos resultados con algunas propuestas planteadas en investigaciones previas vinculadas al tipo de actividades desarrolladas en los distintos contextos espaciales de sitios residenciales y productivos del primer milenio de la Era.

\section{Antecedentes de investigación en el área}

Los restos óseos analizados en este trabajo provienen de las excavaciones de los sitios arqueológicos Uchuquita 1, 2, 3 y 4 -emplazados en la localidad de Anillaco- y de Terraza 5, El Chañarcito y Loma de la Puerta -todos ellos ubicados en la localidad de Los Molinos-. A partir de los fechados radiocarbónicos, se determinó que Terraza 5 y los cuatro sitios que conforman la locación arqueológica Uchuquita se ubicarían temporalmente en el primer momento de la secuencia ocupacional, a la vez que El Chañarcito y Loma de la Puerta se encontrarían en un segundo momento, según el modelo planteado por Cahiza (2015). Los fechados (Tabla 1) realizados en estos sitios fueron calibrados en entorno $\mathrm{R}$, empleando el paquete rcarbon y con la curva Shcal20 (Cahiza et al., 2021). 


\begin{tabular}{|l|l|l|}
\hline \multicolumn{1}{|c|}{ Sitio } & \multicolumn{1}{|c|}{$\begin{array}{c}\text { Fechado } \\
\text { AP }\end{array}$} & \multicolumn{1}{c|}{$\begin{array}{c}\text { Calibrado-1 } \\
\text { sigma d.C. }\end{array}$} \\
\hline Terraza 5 & $1630 \pm 80$ & $388-401$ \\
\hline Uchuquita 1 & $1510 \pm 22$ & $586-606$ \\
\hline Uchuquita 2 & $1625 \pm 20$ & $431-479$ \\
\hline Uchuquita 3 & $1760 \pm 50$ & $251-305$ \\
\hline El Chañarcito SI-R3 & $1300 \pm 60$ & $683-746$ \\
\hline El Chañarcito SI-R8 & $1330 \pm 70$ & $663-774$ \\
\hline Loma de la Puerta & $1320 \pm 80$ & $670-774$ \\
\hline
\end{tabular}

Tabla 1. Fechados radiocarbónicos de los sitios analizados.

El sitio Terraza 5 (Figura 2a) se compone de dos recintos subrectangulares adyacentes entre sí y asociados a campos de cultivo aterrazados (Cahiza, 2015). Ambos fueron excavados y se identificó un único componente ocupacional, con un bajo número de restos. Un fechado radiocarbónico dio como resultado $1630 \pm 80$ años AP, 388-401 cal. d.C. (LP - 3124, carbón vegetal) (Cahiza et al., 2018b). Se ha sugerido que los sitios agrícolas de Castro Barros pudieron vincularse a un tipo de ocupación discontinua asociada a prácticas de cultivo, sin la necesidad de un mantenimiento constante, como ocurre en la actualidad (Cahiza, 2015).

Uchuquita, por su parte, constituye una locación arqueológica de escala doméstica en la que se definieron cinco pequeños asentamientos, cuatro de los cuales fueron excavados (Sabatini y Garate, 2017; Sabatini, 2019). Estos están conformados por una serie de espacios habitacionales vinculados entre sí por espacios abiertos (Figura 2b). En Uchuquita 1 se realizó la excavación integral de dos recintos habitacionales, uno de ellos datado en $1510 \pm 22$ años AP, 586-606 cal. d.C. (D-AMS 028048-material óseo), y un sondeo en un sector abierto. Uchuquita 2 está conformado por dos recintos simples, en uno de los cuales se realizó una intervención extensiva, de donde se tomó otra muestra datada en $1625 \pm 20$ AP, 431-479 cal. d.C. (PSUAMS-6445-carbón vegetal). En Uchuquita 3, asimismo, se excavó la totalidad de un recinto residencial fechado en $1760 \pm 50$ años AP, 251-305 cal. d.C. (LP-3259-carbón vegetal). En Uchuquita 4, por último, se optó por la excavación del único recinto identificado (Sabatini, 2019).

Los estudios arqueofaunísticos realizados en trabajos anteriores en esta localidad arqueológica se focalizaron en Uchuquita 1 y 3; a partir de ellos se determinó la presencia mayoritaria de camélidos y de otros taxones más pequeños, como Dasypodidae y Rodentia. Allí se propuso un modelo de consumo de base familiar con escasa desigualdad y un acceso generalizado a las distintas partes anatómicas de los camélidos (Sabatini y Garate, 2017).

El Chañarcito es un sitio conformado por cinco conjuntos de unidades arquitectónicas (Figura 2c) compuestos por patios y habitaciones, emplazados con unas separaciones de entre cinco y veinte metros entre sí (Cahiza, 2015; Cahiza et al., 2017;

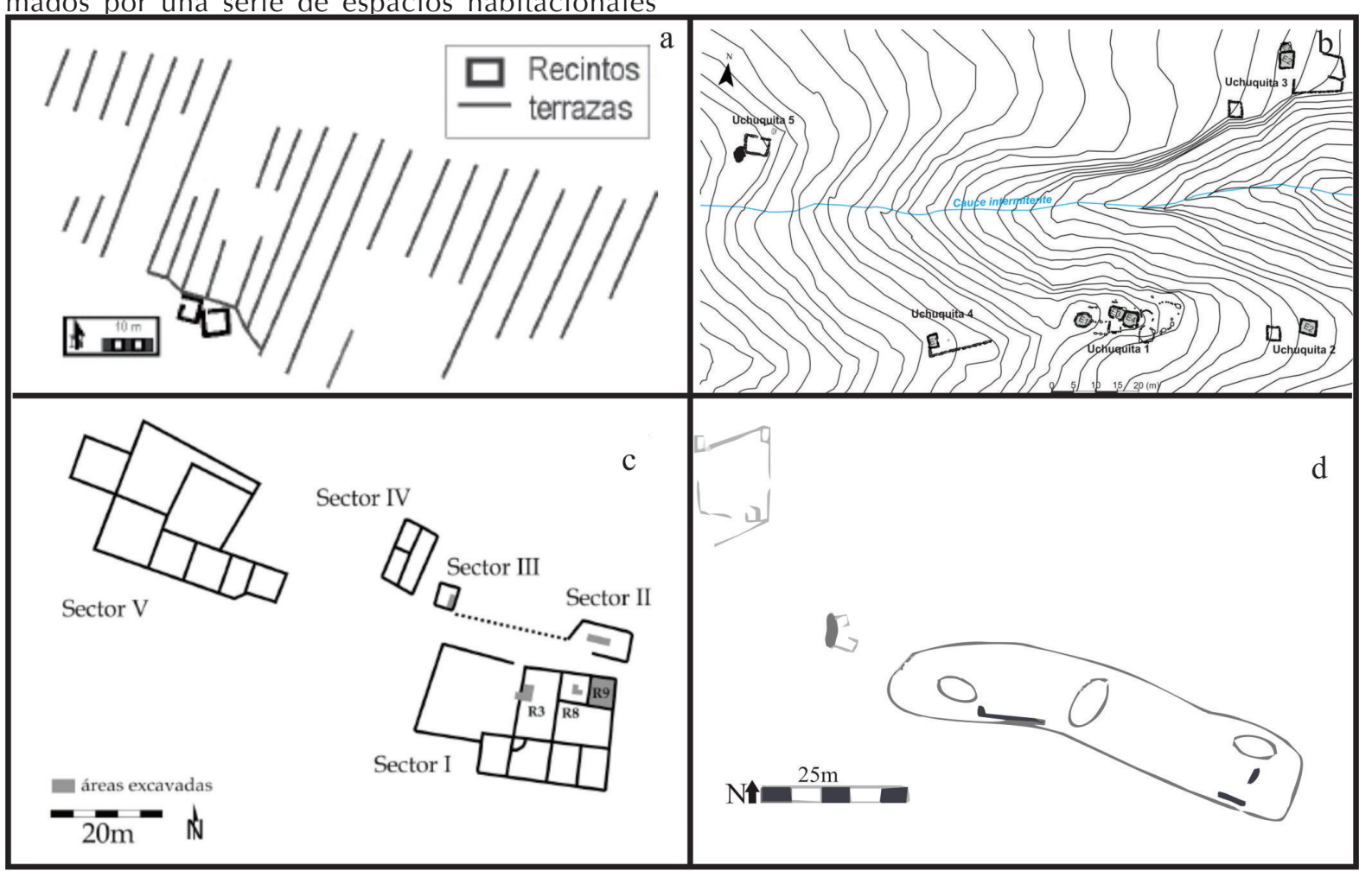

Figura 2. Planos de los sitios estudiados: a) Terraza 5; b) Uchuquita; c) El Chañarcito; d) Loma de la Puerta. 
Cahiza et al., 2018a), en cuyas proximidades se detectaron campos aterrazados de cultivo. En este sitio, de mayor tamaño y complejidad arquitectónica, se excavaron los sectores I, II y III. En el primero de ellos se intervino un patio (Sector I-R3), que presentó una estructura de combustión de la que se extrajo una muestra datada en $1300 \pm 60$ años AP cal. 683 a 746 d.C. (LP - 2952, carbón vegetal). El recinto 8 (SI-R8) constituye una habitación en la que se identificó un enterratorio, cuyo contexto fue datado en $1330 \pm 70$ años AP cal. 663 a 774 d.C. (LP -2959, carbón vegetal). El Recinto 9 (SI-R9), a su vez, fue excavado en su totalidad, y allí fueron identificados un vano con un escalón de acceso desde SI-R8 y una estructura de piedra de combustión rectangular (Cahiza et al., 2017).

El Sector II presenta una plataforma de planta trapezoidal con un lado abierto hacia las unidades domésticas. Este sector de libre acceso y alta visibilidad habría favorecido la interacción social de sus habitantes de forma más fluida que en los demás recintos. A su vez, una rampa con tres escalones que aprovechan el declive natural del terreno ofrece un acceso directo al sitio desde este sector y le otorga cierta jerarquía (Cahiza et al., 2017). Además, se relevó un gran número de fragmentos de cuencos y pucos cerámicos de serie fina para los que se propuso una posible utilidad en contextos de alta visualización, como festividades y demás actos realizados en espacios públicos (Cahiza et al., 2018a).

En el Sector III, por su parte, se intervino parte del Recinto 1, donde se identificó una estructura de piedra que pudo actuar como espacio de almacenamiento (Cahiza et al., 2017). Allí se trazaron dos cuadrículas que fueron excavadas hasta el nivel del suelo natural, a los $80 \mathrm{~cm}$ aproximadamente.

Entre los taxones identificados para el sitio se determinó la presencia de Camelidae, Canidae, Leporidae, Eudromia elegans, Columba livia, Dasypodidae, Rodentia y Aves. Cabe señalar que los camélidos constituyen la categoría más representada en todos los conjuntos. La distribución de los restos relevados arrojó una mayor concentración y diversidad taxonómica en el Sector II, asociado al libre acceso y uso público (Cahiza et al., 2017).

Loma de la Puerta, por último, se emplaza sobre una pequeña loma ubicada en el ingreso a la quebrada del río Los Molinos (Figura 2d). Presenta un muro perimetral de baja altura que delimita la cara sur de la parte superior de la loma, mientras que en el resto del promontorio se observan algunas líneas cortas de muro y cuatro concentraciones de rocas que se corresponderían con estructuras o edificaciones derrumbadas (Cahiza, 2015). Las intervenciones se realizaron en el muro perimetral sur y en una línea de muro y vano de puerta identificado en el sector este. Una muestra fue datada en $1320 \pm$ 80 años AP, 670-774 cal d.C. (LP- 3127, carbón vegetal). Si bien el fechado coincidiría con el período ocupacional de El Chañarcito, la presencia de estilos cerámicos más tardíos y su ubicación en un sector más elevado y próximo al ingreso de una quebrada induce a pensar en posibles ocupaciones posteriores al período 600-800 d.C.

\section{Delimitación de los conjuntos arqueofaunísticos}

Los conjuntos arqueofaunísticos aquí analizados fueron definidos en función de los contextos espaciales de recuperación; de esta manera, se delimitaron nueve conjuntos. El conjunto Terraza 5 (T5) está conformado por la totalidad de especímenes ( $n=20$ ) recuperados en la excavación de los recintos del sitio, los cuales presentaron un único componente ocupacional.

En el caso de Uchuquita, las muestras se organizaron en función de los distintos sitios que corresponden a esta localidad arqueológica. Así se determinaron los conjuntos Uchuquita 1 (U1), Uchuquita 2 (U2), Uchuquita 3 (U3) y Uchuquita 4 (U4), los cuales presentaron un número de restos de $272,55,80$ y 20 , respectivamente.

Para El Chañarcito, los conjuntos fueron discriminados considerando los sectores definidos arquitectónicamente, dado que se han propuesto caracterizaciones funcionales específicas para cada uno de ellos (Cahiza et al., 2018a). Así, fueron agrupados en sector I (SI), en el que se identificaron 602 especímenes óseos; sector II (SII), con 532 restos; y sector III (SIII), en el que se determinó la presencia de 74 especímenes.

Por último, el conjunto Loma de la Puerta (LDP) incorpora la totalidad de restos recuperados para el sitio $(\mathrm{n}=52)$.

\section{MATERIALES Y MÉTODOS}

Para la identificación taxonómica y anatómica se recurrió a atlas osteológicos (por ejemplo, Pacheco Torres et al., 1979) y colecciones osteológicas de 
referencia. En la determinación de especímenes se consideraron las características y rasgos principales de las zonas diagnósticas de cada elemento (Lyman, 1994; Mengoni Goñalons, 2010, entre otros).

Cuando no fue posible determinar si los restos de mamíferos grandes correspondían a Camelidae o Hippocamelus antinensis, se empleó la categoría de Artiodactyla. A su vez, se emplearon categorías más inclusivas en función de la biomasa de los animales vivos (Izeta, 2007) en los casos en que no pudo avanzarse con una identificación taxonómica más precisa. Así, se utilizaron las categorías de Mammalia mediano (MM) para animales de entre 30 y 5 kg; pequeño (MP) para aquellos con pesos inferiores a $5 \mathrm{~kg}$; y simplemente Mammalia (M) para los mamíferos a los que no se les pudo atribuir un peso relativo. Para los taxones pequeños -sean mamíferos o aves-, se empleó la categoría de "pequeño" (P). Finalmente, como "no identificados" (NIT), incluimos las astillas o trabéculas sin identificación posible.

Para cuantificar los conjuntos se consideró el número de restos (NR), identificados o no, y el número de especímenes identificados por taxón (NISP); este último empleado, a su vez, para medir la abundancia taxonómica, y su valor estandarizado (\%NISP) fue utilizado para obtener los índices de utilidad general (IUG) y de secado (IS).

EI IUG para los especímenes identificados de camélidos se obtuvo siguiendo la propuesta de Mengoni Goñalons (2013), quien ofrece una caracterización de elementos en: huesos solo con carne (vértebras, costillas, pelvis y escápulas), huesos con mucha carne y médula (húmero y fémur), huesos con poca carne y médula (radioulna, tibia y mandíbula), huesos solo con médula (metapodios, calcáneo, falanges 1 y falanges 2) y huesos con seso (cráneo).

A su vez, el IS propuesto por De Nigris y Mengoni Goñalons (2005) permite categorizar los restos de camélidos según su potencial de secado en alto (costillas y esternebras), moderado-alto (vértebras y pelvis), moderado-bajo (mandíbula y escápulas) y bajo (huesos largos, falanges 1 y 2 y cráneo), y resulta útil para inferir consumos diferidos (Mengoni Goñalons, 2013).

Se determinó, asimismo, la presencia de rasgos de termoalteración y marcas de origen antrópico (Lyman, 1994; Mengoni Goñalons, 2010), y se consideró la ubicación de dichas marcas en cada elemento para lograr inferir actividades vinculadas al procesamiento, preparación y consumo de alimentos y otros bienes. Entre las marcas no antrópicas, se determinó la presencia de sales, manchas, fracturas, abrasión y roído, y la incidencia de los procesos de meteorización sobre los restos de camélidos y artiodáctilos (Behrensmeyer, 1978).

\section{RESULTADOS DEL ANÁLISIS DE LOS CONJUNTOS FAUNÍSTICOS DE LA SIERRA DE VELASCO}

\section{Terraza 5}

En este conjunto se determinó una composición taxonómica bastante equitativa; los taxones de mayor tamaño (artiodáctilos y camélidos) son los más representados (Tabla 2). Entre los restos de camélidos se identificaron tres fragmentos de costillas, dos con marcas de corte en su cara dorsal, posiblemente vinculadas al proceso de descarne, y dos lumbares. Estos elementos se caracterizan por su alto rendimiento cárnico y alto IS, que los convierte en idóneos para un consumo diferido.

Entre los huesos de Artiodactyla se detectó la presencia de una astilla termoalterada y de un instrumento elaborado sobre un hueso largo con un acanalamiento en torno al sector mesial y un acabado pulido, con desgaste de un extremo, cuya funcionalidad no pudo ser determinada.

Si bien las lumbares de camélidos reflejan estadios de meteorización avanzados (estadio 3), el resto de la muestra presenta un buen estado de conservación general.

\section{Uchuquita 1}

En este caso, se observó una mayor abundancia de Artiodactyla (Tabla 2) como el taxón más representado del conjunto $(n=116)$, seguido por los camélidos $(n=26)$. Es probable que buena parte de los restos de Artiodactyla pertenezcan a camélidos que no pudieron ser identificados con mayor precisión, lo que ampliaría su representación en el registro.

Por su parte, todos los especímenes de dasipódidos corresponden a fragmentos de placas dérmicas, lo que pudo haber incidido en una sobrerrepresentación en la cantidad de individuos (Sabatini y Garate, 2017). Se detectaron ocho de estas placas totalmente quemadas. Asimismo, entre los restos de 


\begin{tabular}{|l|c|c|c|c|c|c|c|c|c|}
\hline & Terraza 5 & \multicolumn{5}{|c|}{ Uchuquita } & \multicolumn{5}{c|}{ El Chañarcito } & $\begin{array}{c}\text { Loma de } \\
\text { la Puerta }\end{array}$ \\
\hline Unidades taxonómicas & T5 & U1 & U2 & U3 & U4 & Sector I & Sector II & Sector III & LDP \\
\hline Camelidae & 5 & 26 & 7 & 8 & - & 36 & 56 & 5 & 9 \\
\hline Artiodactyla & 5 & 116 & 24 & 45 & 11 & 157 & 262 & 37 & 25 \\
\hline Canidae & - & - & - & - & - & - & 1 & - & - \\
\hline Lycalopex sp. & - & - & - & - & - & - & 2 & 1 & - \\
\hline Aves & - & 2 & 1 & - & - & 11 & 8 & 1 & 5 \\
\hline Eudromia elegans & - & - & - & - & - & 1 & - & 1 & - \\
\hline Columba livia & - & - & - & - & - & 1 & - & - & \\
\hline Leporidae & - & 1 & - & - & - & - & 4 & - & - \\
\hline Dasypodidae & - & 23 & 1 & 2 & 5 & 4 & 8 & 1 & - \\
\hline Rodentia & 5 & 33 & 6 & 4 & - & 34 & 6 & 6 & 2 \\
\hline Ranidae & - & - & - & - & - & 1 & 2 & - & 3 \\
\hline Mammalia Mediano & - & 1 & - & - & - & 6 & 24 & - & - \\
\hline Mammalia Pequeño & - & 6 & 3 & - & - & 9 & 11 & 1 & - \\
\hline Mammalia & - & 23 & 6 & 2 & 2 & 39 & 35 & 10 & 1 \\
\hline Pequeño & - & 2 & 1 & - & - & 22 & 5 & 1 & - \\
\hline No identificado & 5 & 39 & 6 & 19 & 2 & 281 & 108 & 10 & - \\
\hline Número de Restos & 20 & 272 & 55 & 80 & 20 & 602 & 532 & 74 & 7 \\
\hline
\end{tabular}

Tabla 2. Los conjuntos óseos de la vertiente oriental de la sierra de Velasco.

aves, un único elemento presentó señales de alteración térmica. En cuanto a la presencia de roedores, si bien su relevancia para el consumo humano no pudo determinarse, sí se comprobó su acción como agentes tafonómicos, lo que se refleja en la presencia de marcas de roído sobre diez diáfisis de huesos largos de Artiodactyla.

Entre los camélidos, las costillas constituyeron los elementos de mayor representación, con un NISP de 11, ocho de las cuales se encontraron quemadas y una con marcas de corte en su sector ventral, actividad asociada a la separación de las costillas de la columna. A su vez, se recuperaron fragmentos de metapodio ( $n=3)$, uno de ellos, quemado; cervicales ( $\mathrm{n}=3$ ); una lumbar quemada; una vértebra; un hueso navicular y un fragmento de primera falange quemados; una epífisis de húmero con una marca de corte asociada al proceso de desarticulación de la pieza; una epífisis de fémur quemada; una diáfisis de tibia quemada y fracturada; un fragmento de tercera falange calcinado y una patella.

EI IUG permite observar una tendencia principal a la explotación de carne (Figura 3) y, en menor medida, al procesamiento de médula. En relación con el IS, se aprecia una tendencia al aprovechamiento de los elementos de mayor potencial para prácticas de secado.
Los procesos de meteorización, por su parte, afectaron escasamente al conjunto; se detectaron solo dos especímenes en estadios 2 y 3 (Behrensmeyer, 1978). Tampoco se apreció un nivel significativo de incidencia de otros agentes naturales.

\section{Uchuquita 2}

En este sector, Artiodactyla $(\mathrm{n}=24)$ y Camelidae $(\mathrm{n}=7)$ vuelven a constituir las categorías más representadas, seguidas por roedores, un fragmento de ave y una placa dérmica de Dasypodidae (Tabla 2).

De los restos de artiodáctilos, un 87,5\% $(n=21)$ se encuentran quemados y calcinados. Entre los camélidos se identificó una costilla con cortes en su extremo distal, una escápula quemada y fracturada, dos fragmentos de radio termoalterados y con cortes (en el sector proximal) y fractura (en el sector distal), una diáfisis de fémur, un navicular y un fragmento de molar. En cuanto a la utilidad de las partes representadas, dos especímenes corresponden a piezas ricas en carne, uno a huesos con mucha carne y médula, y otros dos se caracterizarían por ser huesos con poca carne y médula. Paralelamente, se determinó que el $80 \%(n=4)$ se encuentran entre los huesos de menor potencial de secado (Figura 3). 

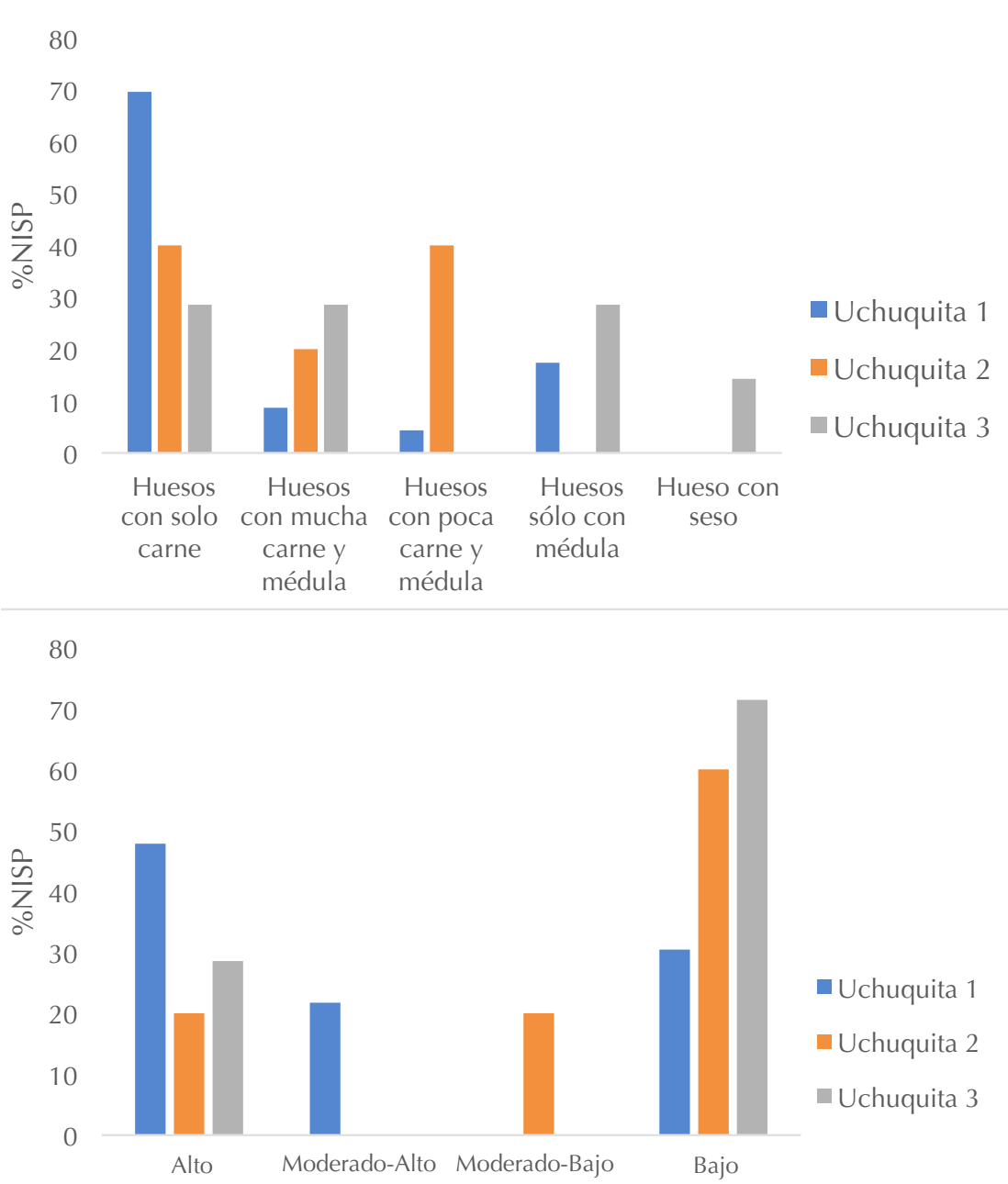

se dividen de forma relativamente equitativa entre restos de bajo y alto índice (Figura $3)$.

\section{Uchuquita 4}

En esta unidad se relevaron solo 11 huesos correspondientes a Artiodactyla (más de la mitad de ellos, quemados), cinco a Dasypodidae y dos a Mammalia. De estos, se comprobó la presencia de seis huesos quemados de Artiodactyla y dos de dasipódidos. No se detectó una marcada incidencia de otros procesos tafonómicos, por lo que se observó un buen estado general de preservación.

\section{El Chañarcito}

En el Sector I, alrededor del $46 \%(n=281)$ del conjunto corresponde a especímenes no identificados (Tabla 2); el recinto 8

Figura 3. Uchuquita 1, 2 y 3. Arriba: Índice de Utilidad General; Abajo: Índice de Secado.

La meteorización no habría incidido en gran medida en la preservación del conjunto; se detectó solo un fragmento de Camelidae en estadio 2 y otro en el estadio 1.

\section{Uchuquita 3}

Aproximadamente el $66 \%(n=53)$ de este conjunto corresponde a Camelidae y Artiodactyla (Tabla 2). Asimismo, se identificaron restos de roedores $(\mathrm{n}=4)$ y dasipódidos $(\mathrm{n}=2)$; se detectó solo una placa dérmica de estos últimos quemada.

Entre los camélidos, se detectaron dos fragmentos de costillas, uno de ellos, quemado; dos diáfisis de fémur quemados y con una fractura helicoidal; dos de metapodios termoalterados, uno de ellos (un extremo articular) con marcas de corte; un fragmento de cráneo y un molar.

Nuevamente, se observa una tendencia al aprovechamiento de elementos con carne por sobre aquellos con médula. Respecto del IS, estos huesos es el que más fragmentos aportó a esta categoría ( $n=206$ ), lo cual se ha explicado por el proceso de remoción sedimentaria realizado durante la inhumación humana en ese espacio (Cahiza et al., 2017). Entre los especímenes identificados taxonómicamente, Artiodactyla y Camelidae constituyen los más representados, seguidos en menor medida por roedores, aves, dasipódidos y ránidos (Tabla 2).

Entre los camélidos, se recuperaron restos de todos los sectores anatómicos excepto la cabeza; los más representados son las costillas $(n=12)$ y los metapodios $(n=8)$, seguidos por fragmentos de vértebras cervicales $(n=3)$, torácicas $(n=2)$ y lumbares $(n=2)$, escápula $(n=2)$, radioulna $(n=2)$, húmero $(n=1)$, tibia $(n=1)$, una pelvis $(n=1)$ de un individuo joven, y una primera $(n=1)$ y segunda falanges $(n=1)$.

Asimismo, se identificaron cuatro instrumentos sobre restos de Artiodactyla. Uno fue elaborado sobre una costilla y, por las dimensiones de su epífisis, parece corresponder a un camélido joven. Aquí se observa la intención de formatizar el hueso 
mediante el desbaste de la parte distal de la cara anterior, en la cual se observa un alisado intencional; es posible que se trate de la fabricación de algún tipo de "espátula" (Pascual Benito, 1998). Respecto de los otros instrumentos, no fue posible realizar una asignación anatómica, dado su grado de formatización.

Por otra parte, se determinó que 14 especímenes de Camelidae y 66 de Artiodactyla presentaron signos de alteración térmica. Asimismo, 17 especímenes de Camelidae poseen marcas de corte; la mayoría de ellas ubicadas sobre diáfisis de huesos largos, especialmente en húmero, radioulna y metapodio. Además, se determinaron cortes sobre una primera y segunda falanges, lo que indica desarticulación y limpieza de piezas. Conjuntamente, se observaron 27 especímenes de Artiodactyla con marcas de corte, raspado, pulido y fracturas intencionales. Entre los demás taxones identificados, se observaron señales de termoalteración sobre restos de roedores $(n=5)$, aves $(n=2)$ y dasipódidos $(\mathrm{n}=2)$.

EI IUG arrojó una tendencia al aprovechamiento exclusivo de carne $y$, en menor medida, a la explotación de médula. Por otra parte, el IS presenta una distribución bastante equitativa entre los elementos de mayor y menor índice, que es apenas superior entre aquellos con mejor potencial de secado (Figura 4).

Entre los procesos que afectaron la preservación del conjunto encontramos la acción de roedores (en nueve fragmentos de Artiodactyla) y abrasión sedimentaria. Asimismo, se identificaron estadios avanzados de meteorización sobre tres diáfisis de Artiodactyla, dos de ellos en el estadio 4 y uno en el 3 (Behrensmeyer, 1978).

60
El Sector II (plataforma) brindó la mayor información faunística (Tabla 2). La distribución vertical de los restos relevados permitió corroborar una acumulación de diversos rellenos, sin detectarse un piso consolidado. Se determinó una mayor diversidad taxonómica, con la presencia de Artiodactyla, Camelidae, Lycalopex sp., Leporidae, Dasypodidae, Rodentia, Ranidae y Aves (Tabla 2). Casi la mitad de los restos se encontraron termoalterados. Se detectó además un espécimen de Canidae (Cahiza et al., 2018a).

Se recuperaron 56 especímenes de Camelidae correspondientes a elementos de toda la carcasa animal. A su vez, se identificaron marcas de corte sobre un fragmento de mandíbula, asociado a la desarticulación del cráneo, y en vértebras cervicales, torácicas y lumbares, que reflejarían actividades de descarne, desarticulación y extracción de músculos. Entre los restos del esqueleto apendicular se hallaron marcas de percusión y fractura en un húmero, posiblemente asociadas a la extracción de
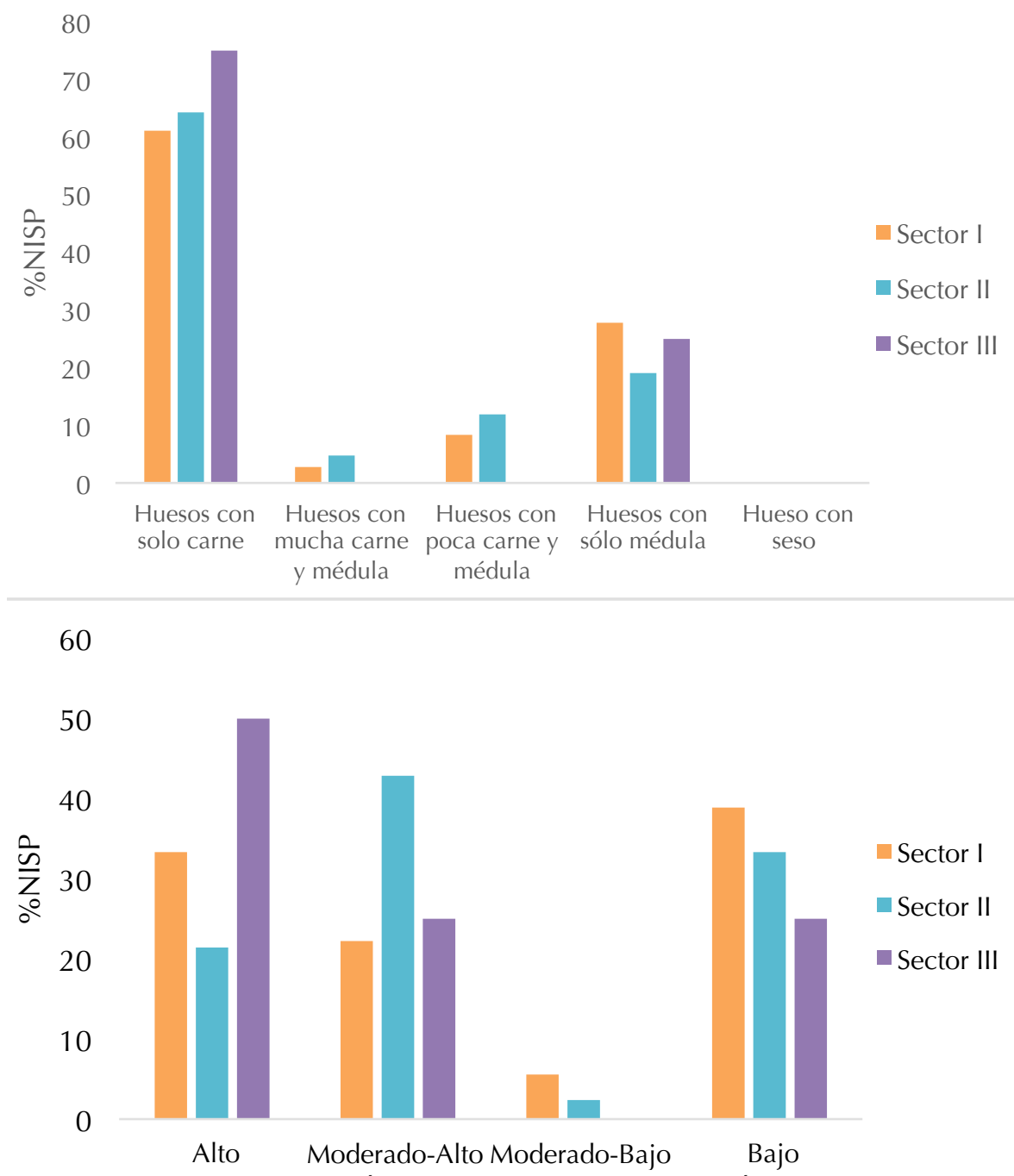

Figura 4. El Chañarcito. Arriba: Índice de Utilidad General; Abajo: Índice de Secado. 
médula; un corte y una fractura en una diáfisis de ulna; y varios fragmentos de metapodios presentaron marcas de corte, fractura y percusión.

Por otra parte, algunos huesos de Artiodactyla presentaron una mayor meteorización respecto de los demás recintos (Cahiza et al., 2017), lo que daría cuenta de pautas de enterramiento diferenciadas en este sector. Asimismo, 12 fragmentos reflejan señales de roído, a la vez que observamos algunos huesos con marcas de abrasión sedimentaria y fracturas naturales.

Nuevamente, el IUG arroja una tendencia al consumo de las piezas de mayor aporte cárnico, a la vez que se confirmó el predominio de restos de alto y moderadamente alto IS, lo cual constituye un posible indicio de consumo diferido (Figura 4).

En SIII se relevó la menor cantidad de restos del sitio (Tabla 2); allí se detectaron camélidos, zorros, martinetas, dasipódidos y roedores. Todos los taxones, excepto Rodentia, presentaron rasgos de termoalteración y marcas de procesamiento. Entre los restos de camélidos, solo se observó un corte sobre una vértebra cervical. Aquí, la meteorización tuvo valores significativos, y se hallaron especímenes de Camelidae $(n=2)$ y Artiodactyla $(n=6)$ en estadios 3 y 4, lo que da cuenta de una mayor exposición previa al enterramiento.

Finalmente, el IUG arrojó una mayor representación de restos con disponibilidad exclusiva de carne, mientras que un $75 \%$ de los especímenes se corresponden con los más aptos para un consumo diferido, aunque el bajo número de huesos impide precisar mejor algún tipo de patrón (Figura 4).

\section{Loma de la Puerta}

En este sitio, los taxones más representados del conjunto corresponden a artiodáctilos y camélidos (Tabla 2). Los restos de Camelidae están conformados por siete fragmentos de costilla (seis de ellos, quemados), un astrágalo (con marcas de corte) y un calcáneo. Las costillas se caracterizan por su utilidad como elementos altamente ricos en carne y con buena disposición para el secado. Por su parte, es factible que el calcáneo y astrágalo pudieran haber ingresado al sitio como elementos acarreados en otras piezas.

A su vez, se determinó que cuatro especímenes reflejaron un grado significativo de meteorización (estadios 2 y 3), mientras que se detectaron marcas de roído solo en dos huesos de Artiodactyla.

\section{DISCUSIONES}

Los análisis faunísticos resultan útiles para contrastar las propuestas funcionales realizadas para los sitios analizados. En Terraza 5, las características arquitectónicas (Cahiza, 2015) y el bajo número de especímenes sugieren una baja intensidad de ocupación. Estudios etnográficos de sociedades agropastoriles del sur andino han demostrado que, en muchos casos, las familias suelen realizar una ocupación temporal de ciertas estancias o puestos vinculados al aprovechamiento estacional de pasturas o áreas agrícolas distantes de la residencia principal (Yacobaccio et al., 1997-1998). Tales prácticas se mantienen vigentes en la actualidad por parte de algunos habitantes de Castro Barros, quienes mantienen activos ciertos espacios agrícolas aterrazados en el piedemonte del Velasco, aprovechando las Iluvias estivales para su irrigación (Sabatini, 2019). El bajo número de restos óseos y su calidad para prácticas de consumo diferido apoyan la hipótesis de un uso temporal y de baja intensidad en el sitio, posiblemente asociado al mantenimiento estacional y uso de las terrazas agrícolas.

En Uchuquita, por su parte, se observaron actividades vinculadas a todas las etapas del procesamiento, lo que sugiere que el ingreso de los animales se habría llevado a cabo de forma integral y que fueron procesados in-situ. La explotación, además, no se restringiría exclusivamente al consumo de carne sino también de médula ósea, y los IS apun$\tan$ a un consumo inmediato tanto en Uchuquita 2 como en Uchuquita 3, mientras que en Uchuquita 1 se aprecia un mayor número de restos con alto índice, lo que posiblemente refleje estrategias vinculadas a la combinación o alternancia de prácticas de consumo inmediato y diferido. La distribución de elementos de cada sector anatómico en los distintos sitios implicaría la ausencia de mecanismos de jerarquización social en el acceso a las diferentes piezas. Todo esto, sumado a las características del registro material y arquitectónico, apoya el modelo de consumo doméstico y familiar planteado en trabajos previos (Sabatini y Garate, 2017; Sabatini, 2019), caracterizado por una tendencia a la explotación de camélidos, aunque incorporando algunos taxones de menor tamaño y de acuerdo con el cual las actividades de procesamiento y consumo fueron llevadas a cabo en la unidad residencial. 
En cuanto al sitio El Chañarcito, se observó una composición taxonómica similar para los tres sectores analizados. Asimismo, mientras se determinó un manejo integral de la carcasa de los camélidos en SI y SII, el SIII presenta un número notablemente inferior de restos y una mayoría de especímenes de camélido de bajo rendimiento. Esto podría deberse a las tareas desarrolladas en cada sector. Para el SIII se ha propuesto un uso vinculado al almacenamiento (Cahiza et al., 2017), lo cual podría resultar en un bajo nivel de consumo, y por tanto, de restos óseos en el recinto. En el Sector II, la significativa acumulación de restos óseos y cerámicos de serie fina (Cahiza et al., 2018a) permite sostener que este sector habría constituido una zona de descarte, que se habría transformado en un segundo momento en un espacio asociado a la reunión y la comensalidad.

Una de las cuestiones discutidas en trabajos previos refiere a la posible desigualdad social en El Chañarcito. Se ha destacado en SI la jerarquización social y simbólica de los recintos R8 y R9, el primero con un enterratorio y el segundo con un acceso restringido y gran cantidad de vasijas de serie fina (Cahiza et al., 2017). A su vez, se ha señalado a las plataformas y montículos como espacio de reproducción de desigualdades (Gastaldi, 2011). Desde una perspectiva microrregional, también se ha enfatizado la relevancia del Chañarcito, al afirmar que en el piedemonte oriental de la sierra de Velasco, "un sitio por cuenca parece tener la jerarquía de centro aldeano con edificaciones ritualizadas" (Cahiza et al., 2018b, p. 41). Estos sitios jerarquizados poseerían una inversión de trabajo que superaría la escala doméstica, lo cual refleja situaciones de labor colectiva y comunitaria.

El análisis arqueofaunístico permite sostener la relevancia de la plataforma como espacio de interacción social, donde los actores sociales (comensales) habrían Ilevado a cabo labores de faenamiento, cocción y consumo de alimentos. Es notable que la realización de reuniones donde los alimentos habrían ocupado un rol destacado y a las que los comensales habrían concurrido con los bienes cerámicos que presentaran mayores esfuerzos de producción por su calidad, estilos y decoración -además de que muchas de ellas expresarían una cosmogonía marcada por un iconografía que refleja figuras felínicas y zooantropomorfas (Cahiza et al., 2018a)- apoyan las ideas plasmadas en trabajos anteriores en las que se sostuvo la existencia de ideas compartidas y prácticas comunitarias.
En el caso de Loma de la Puerta, se reconocieron dos tipos de elementos de camélidos según su utilidad. Por un lado, las costillas (todas termoalteradas) constituyen piezas con alto contenido de carne y, por otro lado, un astrágalo y un calcáneo, elementos de escasa utilidad en relación con el aporte de carne o médula. No se observaron entre los elementos correspondientes al esqueleto axial marcas de procesamiento, aunque sí se precisó una huella de corte sobre el extremo del calcáneo, vinculado al proceso de cuereo y desarticulación de la pieza. Entre los huesos largos de Artiodactyla, la mayoría se encuentran termoalterados y algunos también presentan marcas de corte sobre sus diáfisis, que también estarían vinculadas al proceso de descarne.

De esta manera, se observa una tendencia al consumo de elementos de buen rendimiento cárni$\mathrm{CO}$, y el registro faunístico parece sugerir un ingreso selectivo de piezas al sitio. La presencia de restos de bajo rendimiento se explicaría como elementos acarreados (Binford, 1978) en unidades de mayor rendimiento, cuya separación se habría realizado finalmente en el sitio.

En síntesis, si bien las muestras relevadas hasta el momento son pequeñas, el análisis arqueofaunístico permite señalar algunas tendencias generales. El consumo principal de camélidos y artiodáctilos permaneció en todos los conjuntos, sin marcadas diferencias en relación con la temporalidad o funcionalidad de los sitios. Respecto del aprovechamiento de otros taxones, Rodentia y Dasypodidae constituyeron faunas también representadas en casi todos los sitios analizados, aunque mientras los roedores no suelen presentar rasgos de consumo, gran parte de las placas dérmicas de dasipódidos se encontraron quemadas. La mayor diversidad taxonómica se detectó en El Chañarcito, lo que podría explicarse por la presencia de un área de descarte (sector II) que ofrece una mejor representación de los taxones consumidos, a diferencia de las unidades habitacionales, en donde la limpieza habría jugado un rol importante en la menor acumulación de especímenes óseos.

En cuanto a las estrategias de adquisición o crianza de camélidos, aún no fue posible determinar prácticas de caza o pastoreo. Las excavaciones realizadas hasta el momento han arrojado un número muy bajo de materiales líticos; la ausencia de corrales y la alta fragmentación de los conjuntos óseos dificultó la realización de mediciones osteométricas. 
Por último, la alta fragmentación en la totalidad de las muestras podría estar asociada a los procesos de cocción (reducción de hervido) y a la extracción de médula, aspecto que ha sido señalado para sitios próximos, como en Ambato (Dantas, 2013). Asimismo, no se identificaron indicios de jerarquización a partir de la distribución de partes esqueletarias en los sitios y entre estos, lo que apoya las propuestas de organización comunitarias elaboradas para el área (Cahiza, 2015).

Las diferencias en los conjuntos se derivan principalmente de la funcionalidad de los sitios y de las prácticas realizadas en ellos. De esta manera, es posible observar una continuidad en las prácticas de consumo, al menos para los dos primeros componentes temporales de la secuencia ocupacional del área (300-600 y 600-900 d.C.). Así, se observa un patrón asociado al ingreso general de camélidos a los sitios residenciales de Uchuquita y El Chañarcito, donde fueron procesados y consumidos, realizando un aprovechamiento general de la carne, médula y huesos (para la elaboración de instrumentos).

\section{CONCLUSIONES}

A partir del análisis zooarqueológico de los conjuntos recuperados de sitios de diversa caracterización funcional se evaluaron las tendencias de consumo en el sector nororiental de la sierra de Velasco (departamento de Castro Barros, La Rioja) durante el primer milenio d.C., momento para el cual se observó una recurrencia respecto de los artiodáctilos y camélidos como los taxones más representados en todos los conjuntos. Asimismo, la utilización de índices de utilidad general y secado, y la identificación de sectores anatómicos y de huellas de origen antrópico permitieron aproximarse a las estrategias de adquisición, procesamiento y consumo de recursos faunísticos, a partir de las cuales se definieron áreas de actividad que permitieron contrastar las propuestas funcionales realizadas en trabajos previos a partir de las características arquitectónicas y de los conjuntos cerámicos. Todo esto permitió señalar una continuidad en los patrones de consumo que, al menos, se habría extendido durante los dos primeros componentes temporales de la secuencia ocupacional (300-900 d.C.).

\section{Agradecimientos}

A Pablo Cahiza, Jorge García Llorca, Gabriela Sabatini, Gonzalo García, Cristián Tivani, Sebastián Carosio y Lourdes Iniesta, que colaboraron en los trabajos de campo y laboratorio. Este trabajo fue desarrollado gracias a la beca otorgada por el Consejo Interuniversitario Nacional (año 2017-2018) y gracias al apoyo de la Facultad de Filosofía y Letras (Universidad Nacional de Cuyo) y de CONICET, del Instituto de Ciencias Humanas, Sociales y Ambientales (INCIHUSA-Mendoza).

\section{REFERENCIAS CITADAS}

Behrensmeyer, A. K. (1978). Taphonomic and ecologic information from bone weathering. Paleobiology, 4, 150-162.

Binford, L. R. (1978). Nunamiut ethnoarchaeology. Academic Press.

Cahiza, P. (2015). Un acercamiento espacial a los paisajes comunitarios formativos de Los Molinos -Castro Barros, La Rioja-. Relaciones de la Sociedad Argentina de Antropología, 40(1), 101-122.

Cahiza, P., García Llorca, J., Iniesta, M. L. y Garate, E. (2017). El Chañarcito: Arquitectura, materialidad y consumo de un espacio residencial aldeano de la Sierra de Velasco, La Rioja (CA. 600 al 800 D.C.). Comechingonia. Revista de Arqueología, 21(1), 71-97.

Cahiza, P., Iniesta Di Cesare, L., Sabatini, G. y Ots, M. J. (2018a). Arquitectura y materialidad de la interacción social en la comunidad aldeana del Chañarcito, Los Molinos, La Rioja. Estudios Atacameños, 57, 25-44.

Cahiza, P., Sabatini, L. e Iniesta, M. L. (2018b). Los paisajes sociales del piedemonte nororiental de la Sierra de Velasco, La Rioja (siglo III-IX d. C.). Arqueología, 24(3), 15-33.

Cahiza, P., Garate, E., Sabatini, G., Gheggi, M. S., Iniesta, M. L., Carosio, S. y García, G. (2021). Temporal dynamics of La Rioja village landscape, Argentina. Journal of Archaeological Science: Reports, 39, 103123.

Dantas, M. (2013). Registro faunístico y diferenciación social: el caso de Piedras Blancas, Valle de Ambato, Catamarca (siglos VI-XI d.C.). En A. Izeta y G. Mengoni Goñalons (Eds.), De la Puna a las Sierras: avances y perspectivas en zooarqueología andina (pp. 67- 88). BAR S2564. South American Archaeology Series 19. Archaeopress.

De Nigris, M. y Mengoni Goñalons, G. (2005). The guanaco as a source of meat and fat in the Southern Andes. En J. Mulville y A. Outram (Eds.), The zooarchaeology of fats, oils and dairying, 160-166. Oxbow Books. 
Dlugosz, J. C., Gianfrancisco, M. S., Richard, A., Villar, F. y Núñez Regueiro, V. A. (2009). Arqueología del Sitio El Puesto (Dpto. Castro Barros, La Rioja). Andes, 20, 135-160.

Gastaldi, M. (2011). Cultura material, construcción de identidades y transformaciones sociales en el Valle de Ambato durante el primer milenio d. C. [tesis doctoral. Universidad Nacional de La Plata].

Izeta, A. D. (2007). Zooarqueología del Sur de los Valles Calchaquíes (Provincias de Catamarca y Tucumán, República Argentina): Análisis de Conjuntos Faunísticos del Primer Milenio A.D. BAR International Series 1612. Archaeopress.

Lyman, R. L. (1994). Vertebrate Taphonomy. Cambridge University Press.

Mengoni Goñalons, G. (2010). Zooarqueología en la práctica: algunos temas metodológicos. Xama, 19-23, 83-113.

Mengoni Goñalons, G. (2013). El aprovechamiento de la fauna en sociedades complejas: Aspectos metodológicos y su aplicación en diferentes contextos arqueológicos del NOA. En V. I. Williams y M. B. Cremonte (Eds.), Al borde del imperio, paisajes sociales, materialidad y memoria en áreas periféricas del noroeste argentino (pp. 311-96). Sociedad Argentina de Antropología.

Mercado, G.1993. El yacimiento arqueológico de Anjullón. Investigación Preliminar. Anales de Arqueología y Etnología 48-49, 91-103.

Ortiz Malmierca, M. (2001). "Loma Pircada". Estudios arqueológicos en los faldeos del Velasco. Chuquis, departamento Castro Barros La Rioja (Argentina). Serie Informes de Investigación 2. Agencia Provincial de Cultura.

Outram, A. (2001). A New Approach to Identifying Bone Marrow and Grease Exploitation: Why the "Indeterminate" Fragments should not be Ignored. Journal of Archaeological Science, 28, 401-410.

Pacheco Torres, V., Altamirano Enciso, R. y Guerra Porras, E. (1979). Guía Osteológica para Camélidos Sudamericanos. Serie Investigaciones 4. Departamento Académico de Ciencias Histórico Sociales, Universidad Mayor de San Marcos.

Pascual Benito, J. (1998). Utillaje óseo, adornos e ídolos neolíticos valencianos. Serie de Trabajos Varios del Servicio de Investigación Prehistórica 95. Diputación Provincial de Valencia.

Raviña, G. y Callegari, A. (1988). Mapa arqueológico de la provincia de La Rioja. Revista del Museo de la Plata (Nueva Serie), 9(67), 19-92

Raviña, G. y Callegari, A. (1992). La presencia Aguada en el departamento de Castro Barros (La Rioja). Palimpsesto, 1, 50-70.

Sabatini, G. (2019). Los paisajes aldeanos de la Sierra de Velasco (300-1000 d. C.). Investigaciones arqueológicas de la cuenca de Anillaco, La Rioja [tesis doctoral. Universidad Nacional de Cuyo].

Sabatini, G. y Cahiza, P. (2021). La configuración del paisaje aldeano en Anillaco (La Rioja, Argentina) durante el primer milenio d.C. Intersecciones en Antropología (En este volumen).

Sabatini, G. y Garate, E. (2017). Espacialidad y materialidad de un conjunto de unidades domésticas tempranas -CA. 300-600 D.C.- de Anillaco (La Rioja, Argentina). Comechingonia. Revista de Arqueología, $21(1), 99-122$.

Yacobaccio, H. D., Madero, C. M., Malmierca, M. P. y Reigadas, M. C. (1997-1998). Caza, Domesticación y Pastoreo de camélidos en la Puna Argentina. Relaciones de la Sociedad Argentina de Antropología, XXII-XXIII, 389-418. 